\title{
Can Culling Barred Owls Save a Declining Northern Spotted Owl Population?
}

\author{
Alex Capaldi*, Erin N. Bodine \\ Data collected over the past 25 years has revealed that the Northern Spotted Owl (Strix \\ occidentalis caurina) population of the Oregon Coast region is being displaced by an \\ invasive Barred Owl (Strix varia) population. A component of the present U.S. Fish and \\ Wildlife Service recovery plan is the culling of Barred Owls from Spotted Owl habitat. \\ Using information theory to perform a model selection, we fit the most parsimonious \\ ordinary differential equation species competition model to the data to determine growth \\ rates and competition parameters. We then augment the model to incorporate Barred Owl \\ culling and determine the minimum culling rate required to completely eliminate the \\ Barred Owl population as well as the amount of time required to achieve such an \\ elimination over a range of culling rates. Our analysis shows that even with the culling of \\ Barred Owls, there exists no coexistence equilibrium given the current growth rates and \\ competition parameters, and thus complete elimination of the Barred Owl population is \\ required for the conservation of the Spotted Owl population. Furthermore, the effort \\ required to cull the Barred Owl population is high showing a bleak but not hopeless \\ outlook for this Spotted Owl population. Given the high culling rate necessary, we \\ recommend a robust cost-benefit analysis of the program be conducted of which our \\ study is a first step.
}

